\title{
Síndrome de Austrian en endocarditis infecciosa: reporte de un caso y manejo médico quirúrgico exitoso
}

\author{
Miguel Oyonarte, Andrés Ebensperger, Marcelo Luque, César Del Castillo, Fernando Enrique Lucero, Edgardo Sepúlveda. \\ Centro Cardiovascular, Hospital Clínico San Borja-Arriarán. \\ Trabajo presentado parcialmente en la XLVIII Reunión Anual American College of Physicians, Santiago de Chile.
}

El Síndrome de Austrian, corresponde al cuadro clínico descrito por Robert Austrian en 1957, definido por la triada de Neumonía, Endocarditis Infecciosa (EI) y Meningitis, causado por Streptococcus pneumoniae. En la mayoría de los casos el vicio valvular presente, es la insuficiencia valvular aórtica, cuyo tratamiento médico y resolución quirúrgica de acuerdo con su gravedad, deben ser realizados precoz y oportunamente. Un paciente de 51 años, sin antecedentes de valvulopatía, con historia de poli consumo de alcohol y cocaína comenzó dos semanas previo a su ingreso hospitalario con síndrome febril, neumonía, y meningitis bacteria- na por Streptococcus pneumoniae. Sus hemocultivos fueron negativos. El ecocardiograma transesofágico (ETE) fue compatible con EI valvular aórtica con insuficiencia moderada a severa.

Se trató como EI a microorganismo desconocido y se efectuó un reemplazo valvular aórtico electivo con prótesis biológica a la $5^{\circ}$ semana después de terminado el tratamiento médico antibiótico, cuyo resultado fue exitoso.

Palabras claves: Austrian, síndrome; endocarditis, infecciosa.

\section{Correspondencia:}

Dr. Miguel Oyonarte Gómez,

Santa Rosa 1234, Centro Cardiovascular,

Hospital Clínico San Borja-Arriarán.

miguel.oyonarte@mi.cl 


\section{Austrian Syndrome in Infective Endocarditis}

A syndrome including Infective endocarditis, pneumonia and Meningitis caused by $\mathrm{S}$ pneumoniae was described by Robert Austrian in 1957. The aortic valve is affected in most cases. Medical followed by surgical treatment should be promptly implemented.

The clinical case of a 51 year od man with a history of multiple drug consumption developing fever, pneumonia, and meningitis caused by $\mathrm{S}$ pneumoniae is presen- ted. Blood cultures were negative and trans esophageal echocardiography showed aortic valve vegetations and moderate regurgitation. After multiple antibiotic treatment the patient underwent aortic valve replacement and recovered satisfactorily. Clinical and epidemiological characteristics of this syndrome are discussed. Keywords: Austrian, Syndrome; endocarditis, infective.

\section{Caso Clínico}

Un paciente de 51 años con antecedentes de tabaquismo, poli consumo de drogas y que tenía antecedente de un trauma torácico por herida de bala en hemitórax izquierdo, se presentó con un cuadro de 2 semanas de evolución caracterizado por compromiso del estado general, compromiso cualitativo de conciencia, percepción febril no cuantificada, tos y aumento de volumen con signos inflamatorios en extremidad inferior derecha. Inicialmente, el cuadro se interpretó como síndrome de abstinencia. Por persistencia de la sintomatología, consulta nuevamente en urgencia, donde se interpreta el cuadro como celulitis de pierna derecha y neumonía aspirativa. Se hospitalizó y se inició tratamiento antibiótico en forma empírica con Ceftriaxona asociado a Vancomicina.

Durante la hospitalización progresa el compromiso de conciencia, llegando éste a ser cuantitativo, con GCS 7, Babinski (+) en extremidad inferior izquierda sumado a nistagmus multidireccional y signos meníngeos. Se efectuó una punción lumbar que fue compatible con meningitis bacteriana aguda y en cultivo posterior de LCR se aisló Streptococcus pneumoniae multisensible. Dos hemocultivos fueron negativos y la TAC de encéfalo mostró trombosis del seno transverso y sigmoide izquierdo. Se escaló la terapia, quedando finalmente con Piperaciclina/Tazobactam, Vancomicina, Gentamicina, Corticoterapia y terapia anticoagulante.

Cursó con inestabilidad hemodinámica y falla respirato- ria, fue conectado a ventilación mecánica invasiva, y se usaron drogas vasoactivas.

Por la persistencia del cuadro febril asociado a parámetros inflamatorios elevados, se efectuó un ecocardiograma transesofágico que mostró una válvula aórtica con 3 velos, insuficiencia moderada y 2 imágenes ecogénicas móviles adheridas a cara ventricular de velo coronario derecho y no coronario que impresionaron como vegetaciones.

Se completan 42 días de tratamiento antibiótico, con 4 set de hemocultivos negativos. El ecocardiograma de control mostró una válvula aórtica con 3 velos engrosados en forma significativa, formación sésil en velo coronario derecho que podría corresponder a una vegetación, e insuficiencia aórtica moderada a severa; la fracción de eyección del ventrículo izquierdo fue 60\% (Figuras 1 y 2).

Se decidió la cirugía cardíaca que fue efectuada en el Hospital Clínico San Borja Arriarán. Se objetivó rotura medial en velo no coronario, además de rotura en velo coronario derecho. Se efectuó un reemplazo valvular aórtico con prótesis Perceval L. El paciente evolucionó bien y fue dado de alta al séptimo día postoperatorio.

\section{Discusión:}

Robert Austrian, describió este síndrome en que se asocia la triada de neumonía, endocarditis infecciosa (EI) y meningitis, provocadas por Streptococcus pneumoniae, aunque como él lo señala, el Anátomo Patólogo Austríaco, 

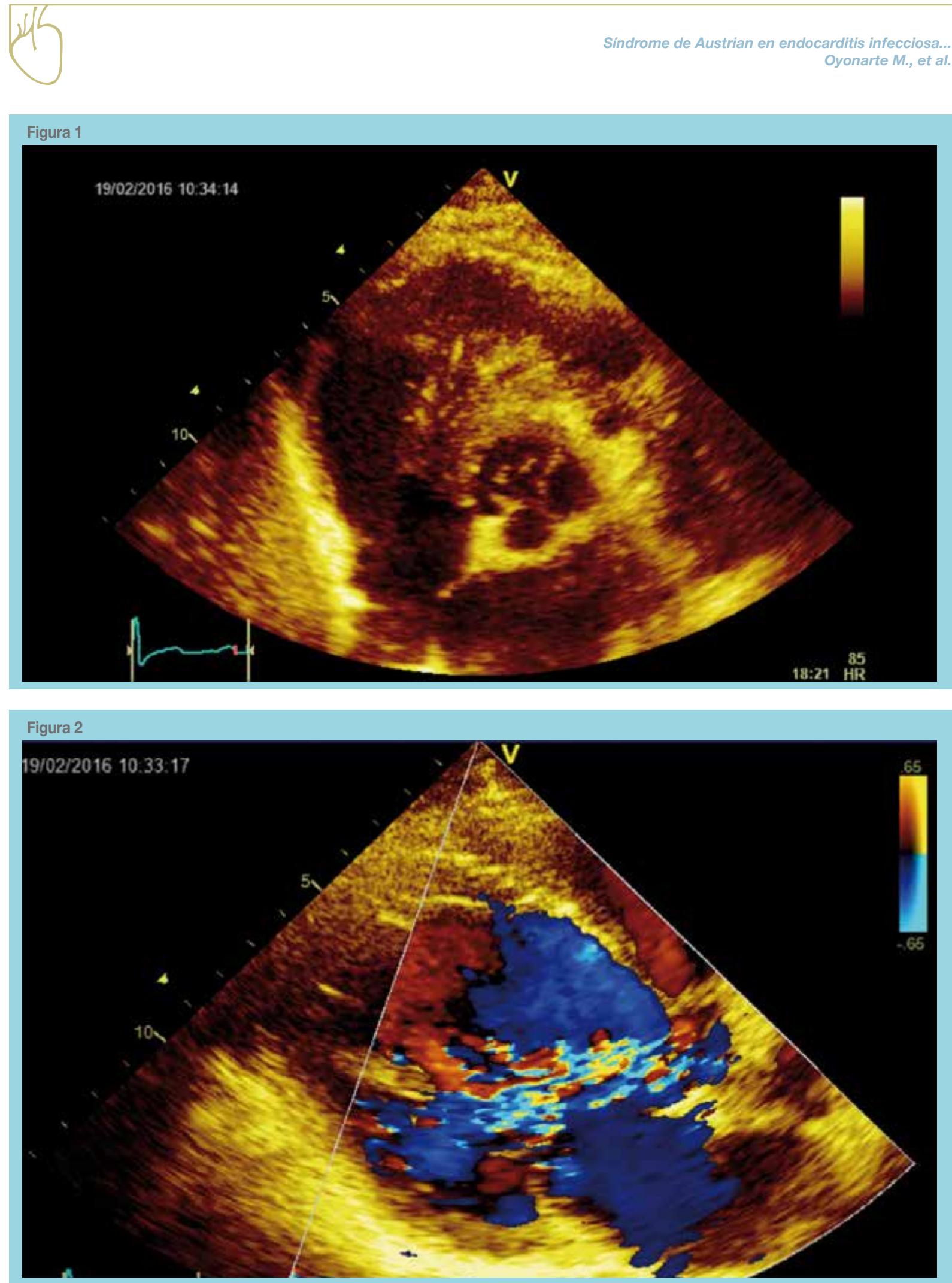

Heschl, describió esta triada en 5 necropsias en $1862^{1,2}$. La infección por Streptococcus pneumoniae ha bajado en incidencia en forma notable. En la era pre antibiótica se encontraba en el 15 a $30 \%$ de las causas de EI, pero en la actualidad esa cifra ha disminuido a menos del $3 \%$ e incluso a $1,2 \%$ en algunas series ${ }^{3-5}$.

En nuestro paciente, la puerta de entrada de este microorganismo fue pulmonar, con probable diseminación hematógena a corazón y luego embolizó a meninges. Se aisló Streptococcus pneumoniae en el líquido céfalo 
raquídeo y el hemocultivo fue negativo. Habitualmente, esta enfermedad afecta a varones y a la válvula aórtica, describiéndose también compromiso original en válvula mitral ${ }^{3,9}$. La asociación de neumonía y meningitis, constituyen una clave epidemiológica que debe, en caso de hemocultivos negativos, hacer sospechar esta entidad, lo que tiene vital importancia para el desenlace ${ }^{6}$, más aún en paciente adictos ${ }^{7}$. El ecocardiograma en la EI, esencialmente el ETE, es indispensable para su diagnóstico y manejo médico quirúrgico ${ }^{8}$. El alcoholismo se encuentra presente en $38 \%$ de los casos y la mayor susceptibilidad en adquirir la infección se explicaría por una respuesta leucocitaria disminuida, con una defectuosa quimiotaxis y fagocitosis ${ }^{10}$.

El tratamiento quirúrgico, idealmente, debe ser precoz, y ha reducido la mortalidad en estos pacientes ${ }^{10,11}$.

La válvula Perceval, está validada como una prótesis bio- lógica estándar en los pacientes que requieren un reemplazo valvular biológico. En nuestro grupo esta válvula se está empleando como primera opción para reemplazo valvular biológico, por sus ventajas en términos de fácil instalación y buen perfil hemodinámico en el postoperatorio.

En la revisión efectuada por Kanakadandi et al ${ }^{10}, 19$ de 31 pacientes requirieron cirugía de reemplazo valvular. La mortalidad global de la serie fue $22 \%$, similar al $20 \%$ reportado por González-Juanatey ${ }^{7}$, que es también similar a la observada en EI por S. pneumoniae ${ }^{12}$.

El esquema antibiótico empleado en este paciente fue a microorganismo desconocido: Piperaciclina/Tazobactam, Vancomicina y Gentamicina; que posteriormente se desescaló a Ceftriaxona y Gentamicina. La cirugía se efectuó en forma electiva, 5 semanas después de completado tratamiento médico antibiótico.

\section{Referencias}

1- AUSTRIAN R. Pneumococal endocarditis, meningitis and rupture of the aortic valve. Arch Intern Med 1957; 99: 539 - 44.

2- HESCHL. Pathologisch-anatomische mittheil- ungen aus dem gratzer allgemeinen kranken - house: 4 . Zur casuistic und aetiologie der endocarditis (fortsetzung), Oester. Ztschr. Pract. Heilk. 1862; 8: 238.

3- RAHIM MA, ZAMAN S, FAHMIMA H, RAHAT S, NAZIM K. Succesful medical management of a case of austrian síndrome - an uncommon entity in the modern antibiotic era: a case report. BMC Res Notes 2017; 10: 456.

4- GRACIA BC, ESCALANTE B, VELILLA C. Síndrome de austrian. Rev Clin Esp 2016; 6: 338.

5- LEFORT A, MAINARDI JL, SELTON-SUTY C, CASASSUS P, GUILLEVIN L, LORTHOLARY O. Streptococcus pneumoniae endocarditis in adults. a multicenter study in france in the era of penicillin resistance $(1991-1998)$. The pneumococcal endocarditis study group. Medicine (Baltimore) 2000; 79: 327 -37 .

6- BADDOUR L, FREEMAN W, SURI R, WILSON WR. "Infecciones cardiovasculares". En braunwald heart disease. A text book of cardiovascular medicine. Edición española de la $10^{\circ}$ ed. 2016. Elsevier España SLU, 1541.
7. GONZÁLEZ- JUANATEY C, MAYO J, TESTA A, GONZALEZ-GAY M. AUSTRIAN SYNDROME: Report of two new cases and literature review. International Journal of Cardiology 2006; 108: 276 - 275.

8. JACOB S, TONG T. Role of echocardiography in the diagnosis and management of infective endocarditis. Curr Op Cardiol 2002; $7: 474-85$.

9. ECHEVERRI D, VARGAS M, MATTA L, ROSSO F, SEGURA J. Infección invasiva por streptococcus pneumoniae: reporte de un caso de un paciente con síndrome de austrian. Biomédica $2015 ; 35: 16$ - 20.

10. KANAKADANDI V, ANNAPUREDDY N, AGARWAL S. K. et al. The austrian sindrome: a case report and review of the literature. Infection (2013) 41: 695-700.

11. ATKINSON K., AUGUSTINE DX, EASAW J. Austrian syndrome: a case report and review of literature. BMJ Case Rep. 2009; bcr03.2009.1724.

12. MARTÍNEZ E., MIRÓ J. M., ALMIRANTE B., et al. Effect of penicillin resistance of streptococcus pneumoniae on the presentation, prognosis and treatment of penumococcal endocarditis in adults. Clin Infect Dis 2002: 35: 130-9 